\title{
Stiffness Analysis of Reinforced Ureteral Stents Against Radial Compression: In vitro Study
}

This article was published in the following Dove Press journal:

Research and Reports in Urology

\author{
Benoît Vogt $\mathbb{D}$ \\ Department of Urology, Polyclinique de \\ Blois, La Chaussée Saint-Victor 4I260, \\ France
}

Introduction: Malignant ureteral obstruction caused by cancer diseases may induce renal failure. Indwelling stent is a popular method to release renal obstruction. But adequate stent placement across an obstructed ureter does not necessarily guarantee renal decompression. The aim of the study was to compare, in vitro, the physical characteristics and stiffness of several commercially available reinforced ureteral stents and identify the physical factors that could lead to the obstruction of the stent.

Material and Methods: The test apparatus used for measurements allowed applying a radial compression force on a segment of the stent to stop a water flow through the lumen of the stent. Some reinforced double-pigtail stents Teleflex Medical, Bard, and Coloplast were evaluated.

Results: The best physical-stiffness characteristic was obtained with the Teleflex $8 \mathrm{~F}$ stent $\left(5.4 \mathrm{~N} \mathrm{~mm}^{-2}\right.$ ). The best result against the radial compression was obtained with tandem stents. The radial compressive stresses of the Teleflex stents $\left(4.4\right.$ to $5.4 \mathrm{~N} \mathrm{~mm}^{-2}$ ) were higher than with the other stents used in the study $\left(1.0\right.$ to $2.9 \mathrm{~N} \mathrm{~mm}^{-2}$ ). Among the reinforced stents selected in the present study, a wider inner diameter helped increase volumetric flow rate but did not affect the stiffness of the stent. The measurement of inner diameter showed heterogeneity along the tube of some stents.

Conclusion: The stiffness of the stent appeared to be an important factor to maintain patency with respect to radial compression forces but the inner diameter of the stent and its preservation may be essential parameters to increase the volumetric flow rate. Some reinforced stents tested in the present study confirmed that it is possible to combine stiffness and wide lumen. The use of tandem stents provided the best stiffness against radial compression and the greatest lumen.

Keywords: ureteral stent, stiffness, diameter, stent, compression, malignant ureteral obstruction

\section{Introduction}

Malignant ureteral obstruction (MUO) caused by cancer diseases is not an infrequent situation and may induce renal failure, renal colic or pyelonephritis. Chronic renal insufficiency is a barrier to several therapies including chemotherapy. Indwelling stent is a common method to release renal obstruction. ${ }^{1,2}$ But adequate stent placement across an obstructed ureter does not necessarily guarantee renal decompression. Thus, the urologist must be aware of the relatively high rate of stent obstruction in patients with MUO. Most studies reported an approximately $40 \%$ failure rate which constitutes a challenge in the management of malignant diseases. ${ }^{1-3}$ These obstructions involved a complex system that incorporates severity of the cancer disease, stent properties,
Correspondence: Benoît Vogt Department of Urology, Polyclinique de Blois, I Rue Robert Debré, La Chaussée Saint-Victor 4I260, France

Tel +332549065 I I

$\mathrm{Fax}+33254906566$

Email message@benoitvogt.fr
Research and Reports in Urology 2020:12 583-59|

cc) (1) 2020 Vogt. This work is published and licensed by Dove Medical Press Limited. The full terms of this license are available at https://www.dovepress.com/terms.php and BY NC incorporate the Creative Commons Attribution - Non Commercial (unported, v3.0) License (http://creativecommons.org/licenses/by-nc/3.0/). By accessing the work you hereby accept the Terms. Non-commercial uses of the work are permitted without any further permission from Dove Medical Press Limited, provided the work is properly attributed. For permission for commercial use of this work, please see paragraphs 4.2 and 5 of our Terms (https://www.dovepress.com/terms.php). 
compressive forces created by the MUO, development of encrustation and colonization on the ureteral stent. ${ }^{1,4,5}$ The stiffness of the stent appeared to be an important factor to maintain patency with respect to compression forces. ${ }^{1,3,4}$

In order to reduce drainage failures, a useful tool would be to provide practising urologists information that may help them select a more appropriate stent if the stent currently in place is obstructed. From June 2009 to June 2019 in a single institution, 150 consecutive patients requiring indwelling stent for MUO were fitted with commercially available reinforced ureteral stents. The progressive choice of stents was shaped by a single surgeon (BV) to overcome each failure. The aim of the study was to compare, in vitro, the physical characteristics and stiffness of each stent used during this period and, identify the physical factors that could lead to the obstruction of the stent.

\section{Materials and Methods}

The test apparatus used in the present study for measurements was designed and assembled de novo to reproduce tumor radial compression on the stent (Figure 1). The tool was entirely made of metal, allowing a force of $2.9 \mathrm{kN}$ to be applied by means of a worm screw between 2 plates $25 \mathrm{~mm}$ wide and $2 \mathrm{~mm}$ thick. Two digital force transducers of different sensitivities were used (Handifor $50 \mathrm{~kg}$, $0.5 \%$ and WeiHeng WH-C $300,300 \mathrm{~kg}$ ).

For the test, a new $5 \mathrm{~cm}$-long-segment stent was inserted between the plates and the volumetric flow rate was measured with a pressure of $20 \mathrm{~cm}$ water. The initial water velocity was determined by dividing the volumetric flow rate by the cross-sectional area. The kinetic water velocity was estimated during radial compression by analogic recording of the variations of a horizontal infrared beam perpendicular to the direction of the stent. When necessary, the holes of the perforated stents were sealed with a soft tape. The worm screw was used by manually screwing it to compress the stent between the metal plates. The maximal force reading was recorded for each trial when the water flow disappeared. This process was repeated at least five times for each stent with water at room temperature. The results of the compression forces were in Newton $(\mathrm{N})$. The uniaxial stress, which is the force causing the deformation divided by the unit area to which the force is applied, was assimilated to a specific characteristic of each stent and was in $\mathrm{N} \mathrm{mm}^{-2}$. Infrared sensors (Universal LED Infrared Emitter and Receiver Diode; 940nm; 5mm; $3.3 \mathrm{~V}$ ) and Elegoo card
UNO R3 ATmega328P Board were used for electronic testing.

Double-pigtail stents without holes such as Teleflex Medical Tumor Stents (6F [named T6], 7F [T7], 8F [T8]), Bard Angiomed Urosoft Tumor Stents (7F [B7], 8F [B8]), Coloplast Vortek Tumor Stents (7F [P7TS], 8F [P8TS]) and, Coloplast Silicone Tumor Stent (7F [S7TS]) were evaluated for radial compression. Other stents with holes, not currently used in tumor drainage, were also tested to account for a stiffness scale as Coloplast Vortek (7F [P7], 8F [P8]). The outer and inner diameters in the middle of the stent were provided by the manufacturers. A manual measurement of the inner diameter by successive introduction of catheters with determined diameters was carried out at different levels of the stent.

Statistical Analysis. The data are presented as mean \pm SD. Data were analysed using the Wilcoxon-Mann-Whitney test. Values of $p<0.05$ were considered significant.

\section{Results}

Physical forces, stresses, volumetric flow rate and diameters of stents used in the study are reported in Tables 1 and 2. The best physical-stiffness characteristic was obtained with the Teleflex $8 \mathrm{~F}$ stent $\left(5.4 \mathrm{~N} \mathrm{~mm}^{-2}\right)$. The best result against the radial compression was obtained with a tandem of two Teleflex $8 \mathrm{~F}$ stents offering a double lumen and requiring a double force $(731.8 \mathrm{~N})$ to stop the water flow. The radial compressive stresses of the Teleflex stents (4.4 to 5.4 $\mathrm{N} \mathrm{mm}^{-2}$ ) were higher than with the other stents used in the study $\left(1.0\right.$ to $\left.2.9 \mathrm{~N} \mathrm{~mm}^{-2}\right)$. The reinforced stents were all significantly different for stresses and forces when considered in pairs.

Among the reinforced stents selected in the present study, a wider inner diameter helped increase the volumetric flow rate but did not affect the stiffness of the stent (Table 2 and Figure 2).

The analysis of the kinetics of water velocity in all stents tested showed a progressive increase at the beginning of the compression then a sudden drop until the disappearance of the water flow (Figure 3).

The measurement of inner diameters showed heterogeneity along the tube for the $\mathrm{B} 8$ with inner diameter narrowing to $1.20 \mathrm{~mm}$ at the ring, for P8TS and P7TS with inner diameter narrowing to $1.0 \mathrm{~mm}$ at the tightening near the bladder loop and, for T7 and T6 with inner diameter narrowing to $1.3 \mathrm{~mm}$ and 1.0 at the junction between the reinforced tube and the loop, respectively (Table 2). 


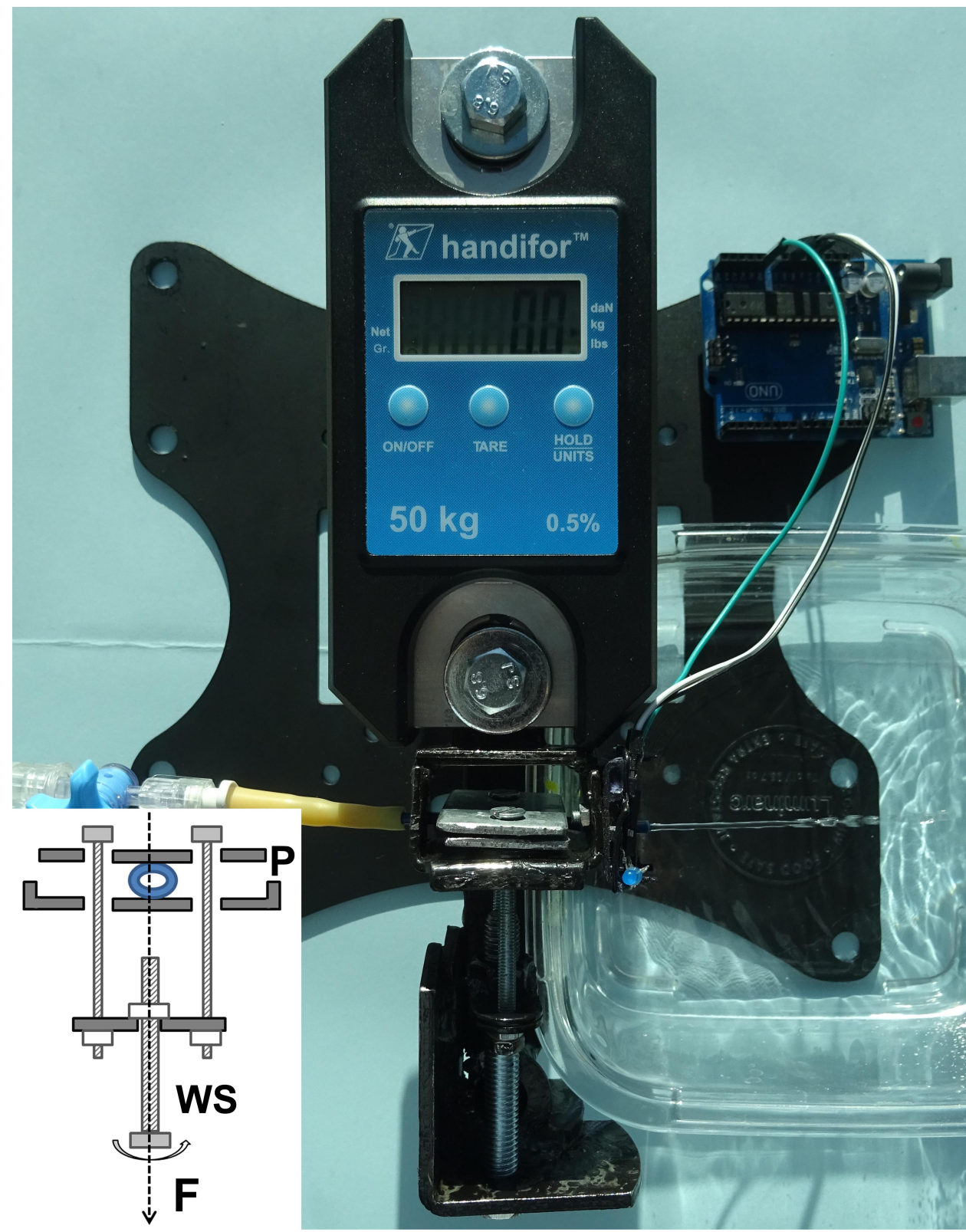

Figure I The test apparatus used for measurements was entirely made of metal and designed to reproduce radial compression on the stent until the water flow disappears. The rotation of the worm screw (WS) was used to apply an evenly-spread force (F) between the two metal plates (P).

\section{Discussion}

\section{Study Design}

The ureteral stents are defined as devices that are inserted inside obstructive ureters to maintain renal patency. With MUO, renal failure occurs when luminal vacuity of the ureter disappears because no flow between the stent and the ureter wall is possible. Obstruction of a ureteral stent occurs with complete disappearance of the internal lumen by crushing or encrustation. ${ }^{5}$ The study design with a measurement of the radial compression leading to the disappearance of the water flow was therefore close to clinical reality. As the determination of the disappearance of the water flow may be subjective, the tests were repeated using the transmission of ultrasound through the lumen of the stent. The sudden disappearance of ultrasound was recorded and the results obtained with ultrasound confirmed the results obtained with the water flow. As the results did not provide any new information, they have not been reported in the tables.

Assuming that the diuresis is approximately $1500 \mathrm{~mL}$ per day, the ureteral flow rate on one side is approximately $0.5 \mathrm{~mL} \mathrm{~min}^{-1}$. This flow rate is too low to analyze the 
Table I Outer Diameters, Compression Forces and Stresses of Stents Used in the Study. The Reinforced Stents Were All Significantly Different for Stresses and Forces When Considered in Pairs $\left(*_{p}<0.05\right)$

\begin{tabular}{|l|l|l|l|l|}
\hline & $\begin{array}{l}\text { Outer Diameter } \\
\text { (French) }\end{array}$ & $\begin{array}{l}\text { Outer Diameter } \\
(\mathbf{m m})\end{array}$ & $\begin{array}{l}\text { Stress for Complete Obstruction } \\
\left(\mathbf{N ~ m m} \mathbf{~ m m}^{-\mathbf{2}}\right.\end{array}$ & $\begin{array}{l}\text { Compression Force for Complete } \\
\text { Obstruction (N) }\end{array}$ \\
\hline Tandem & 8 & 2.67 & $5.4 \pm 0.1^{*}$ & $731.8 \pm 1.8^{*}$ \\
T8 & & 2.67 & $5.4 \pm 0.1^{*}$ & $362.9 \pm 9.8^{*}$ \\
T8 & 8 & 2.33 & $4.4 \pm 0.1^{*}$ & $257.0 \pm 4.3^{*}$ \\
T7 & 7 & 2.00 & $4.4 \pm 0.3^{*}$ & $217.7 \pm 16.5^{*}$ \\
T6 & 6 & 2.67 & $2.9 \pm 0.1^{*}$ & $190.1 \pm 6.0^{*}$ \\
B8 & 8 & 2.33 & $2.7 \pm 0.1^{*}$ & $156.9 \pm 6.9^{*}$ \\
B7 & 7 & 2.67 & $1.4 \pm 0.1^{*}$ & $94.2 \pm 8.7^{*}$ \\
P8TS & 8 & 2.33 & $1.4 \pm 0.2^{*}$ & $83.7 \pm 11.6^{*}$ \\
P7TS & 7 & 2.33 & $1.0 \pm 0.1^{*}$ & $58.4 \pm 3.6^{*}$ \\
S7TS & 7 & 2.67 & $0.9 \pm 0.1$ & $62.2 \pm 3.8$ \\
P8 & 8 & 2.33 & $1.7 \pm 0.1$ & $98.5 \pm 4.0$ \\
P7 & 7 & &
\end{tabular}

Table 2 Volumetric Flow Rate at $20 \mathrm{~cm}$. Water for a $5 \mathrm{~cm}$-Long-Segment Stent, Manufacturer Diameters and Diameters by Manual Measurement of Stents Used in the Study

\begin{tabular}{|l|l|l|l|l|}
\hline & $\begin{array}{l}\text { Volumetric Flow Rate at } \\
\mathbf{2 0} \mathbf{c m} \text { Water }\left(\mathbf{m L} \mathbf{~ m i n}^{-1}\right)\end{array}$ & $\begin{array}{l}\text { Inner Diameter } \\
\text { by } \\
\text { Manufacturers }\end{array}$ & $\begin{array}{l}\text { Tubular Constriction Zone } \\
\text { Diameter by Manual Measurement } \\
(\mathbf{m m})\end{array}$ & $\begin{array}{l}\text { Inner Diameter without } \\
\text { Sleeve by Manual } \\
\text { Measurement (mm) }\end{array}$ \\
\hline Tandem & - & $1.60 \pm 0.1$ & 1.55 & - \\
T8 & $104.2 \pm 1.0$ & $1.60 \pm 0.1$ & 1.55 & - \\
T8 & $104.5 \pm 1.0$ & $1.60 \pm 0.05$ & 1.30 & - \\
T7 & $1.30 \pm 0.05$ & 1.00 & - \\
T6 & $70.6 \pm 0.6$ & $1.30 \pm 0.04$ & 1.20 & 1.65 \\
B8 & $64.1 \pm 0.4$ & $1.00 \pm 0.02$ & 1.10 & 1.45 \\
B7 & $34.8 \pm 0.3$ & 1.08 & 1.00 & 1.55 \\
P8TS & $46.7 \pm 1.0$ & 1.08 & 1.00 & 1.55 \\
P7TS & $43.1 \pm 0.3$ & 1.00 & - & - \\
S7TS & $34.6 \pm 0.3$ & 1.53 & - & - \\
P8 & $87.2 \pm 1.1$ & 1.25 & - & - \\
P7 & $62.4 \pm 0.5$ &
\end{tabular}

interruption of the water flow in the stent by radial compression. The pressure of $20 \mathrm{~cm}$ water provided a flow rate of around $80 \mathrm{~mL} \mathrm{~min}^{-1}$ and makes it possible to analyze the flow through the stent. With $20 \mathrm{~cm}$ water, the water pressure at the inlet to the stent was $2 \mathrm{kPa}$ while the pressures obstructing the stents ranged from 1 to $5 \mathrm{MPa}$ (or 1 to $5.10^{6} \mathrm{~N} \mathrm{~m}^{-2}$ ). The water pressure was therefore much lower than the compressive pressures and could not in itself consist of radial compressive strength. Thus, stresses were only related to the characteristics of the stent.

\section{Stiffness}

Ureteral stents are anisotropic tubes with varying outer and inner diameters, different chemical compositions and various structures (sleeve for B8 and P8TS or wire mesh for T8). Christman et al previously reported that the physical structure of the stent was damaged by the compression area, indicating that the elastic limit was exceeded. ${ }^{4}$ The characterization of the radial compression must therefore be limited to the force applied in Newton or to the stress which distributes the force per unit area in $\mathrm{N} \mathrm{mm}^{-2}$. In addition, the stress is a good means to reflect the weight to be applied on each $\mathrm{mm}^{2}$ of the compressed segment of the stent and achieve complete obstruction. For example, $540 \mathrm{~g}$ on each $\mathrm{mm}^{2}$ of the T8 was needed to stop the water's flow, while only $100 \mathrm{~g}$ was sufficient for the S7TS (silicone stent).

The best physical-stiffness characteristic was obtained with the Teleflex 8F stent (Table 1). The Teleflex 8F (T8) 


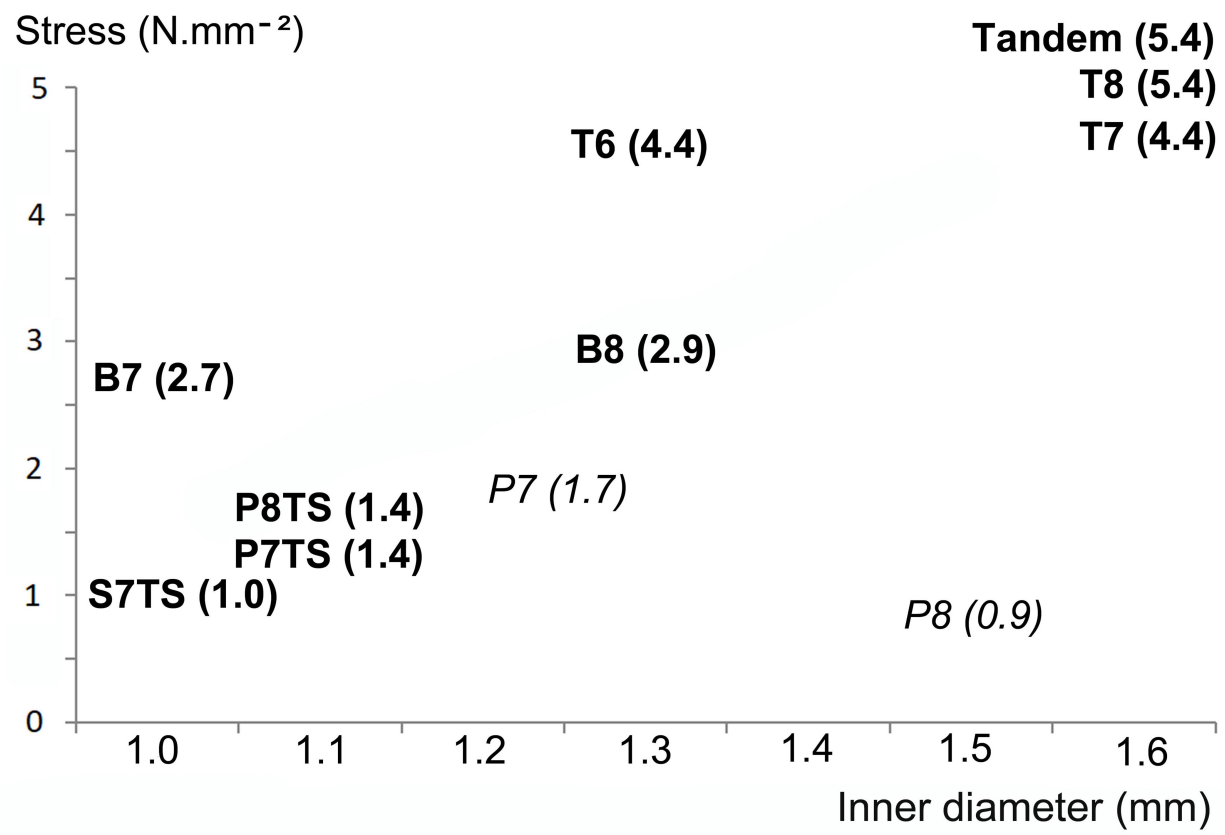

Figure 2 Stresses of the stents according to the inner diameter showing that among the selected stents, a wider inner diameter did not affect the stiffness of the stent (reinforced stents in bold, standard stents in italic and, stresses in parentheses).

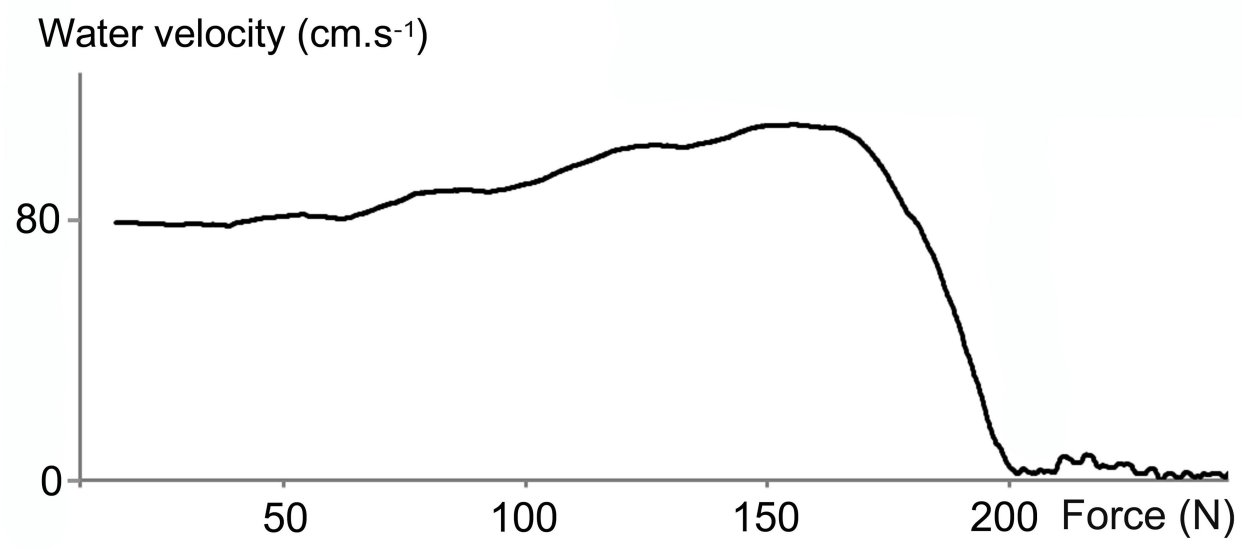

Figure 3 Kinetics of water velocity in the Tumor Stent Bard 8F (B8) during radial compression. The recorded data showed a progressive increase in velocity at the beginning of the compression then a sudden drop until the disappearance of the water flow.

was armed with a rigid wire mesh while a sleeve was slipped into the B8 and P8TS. The polyimide sleeve of the $\mathrm{B} 8,{ }^{6}$ which was more rigid than that of the P8TS, may explain the difference in stiffness. In contrast, among the reinforced stents, the S7TS silicone stent had the lowest stiffness. Silicone material is known to be soft. In a series of patients who had undergone percutaneous placement of a nephrostomy tube and double-pigtail stent, antegrade pressure-flow studies were performed by Hübner et al. The authors noted that an increased flow resistance was observed with the $7 \mathrm{~F}$ silicone stent and when $5 \mathrm{~F}$ or $6 \mathrm{~F}$ silicone stents were kinked, the flow disappeared. ${ }^{7}$ Interestingly, the P7 appeared to be stiffer than the P7TS or P8TS. This difference could be explained by the fact that the P7TS and P8TS have a $1.55 \mathrm{~mm}$-wide lumen without their sleeve and therefore have a wall easier to compress.

In a previous study, Christman et al measured the force needed to compress the stent to $50 \%$ of its original external diameter. With regard to the inner diameters, this stress on the wall would have resulted in the complete obstruction of the stent. The forces observed were lower (12 to 
$144 \mathrm{~N}$ ) because the compression area was probably less extensive than in the present study. He also observed differences in stiffness between the stents according to the external diameters and the composition of the stents. ${ }^{4}$

\section{Diameter}

Among the reinforced stents selected in the present study, the stiffness of the stent increased with the inner diameter of the stent. This apparent increase resulted from the management of clinical failures with a deliberate choice of stents, combining wide diameter and stiffness. However, Christman et al observed that some 7 and $8 \mathrm{~F}$ stents had the greatest inner diameter but low stiffness. ${ }^{4}$ In the study, the inner diameter of P8 was wide but the stiffness was low at $0.9 \mathrm{~N} \mathrm{~mm}^{-2}$. The P8 easily obstructed by compression force was not a reinforced stent and therefore was not compared to other reinforced stents. In contrast, some reinforced stents tested in the present study confirmed that it is possible to combine stiffness and wide lumen (Figure 2).

The urine flow through a stented ureter has already been studied in several ways. With physiological flow rates and without ureteral obstruction, Hübner et al found that even small-diameter stents drain sufficiently. ${ }^{7}$ By using a mechanical ureteral model, Stoller et al even indicated that the stent lumen could be completely occluded if the extraluminal space was large enough. ${ }^{8}$ Kim et al investigated urine flow by computational fluid dynamic simulations and observed that the total flow rate in the ureter with an in-stent stenosis did not show any difference with the total flow rate in the stented ureter because the decrease in luminal flow rate was compensated by an increase in extraluminal flow rate. ${ }^{9}$ Finally, the role of the side holes in the passage of the flow in and around the stent was enhanced around a stenosis. ${ }^{9,10}$

However, there is no side hole along the tube of the reinforced stent and the extraluminal space disappears in MUO. Then, urine flow can take place only through the lumen. Another mechanism of stent failure was occlusion of the stent lumen by debris that may be produced in direct response to the presence of the stent ${ }^{4}$ and, if the stent was partly compressed it takes only a minimal amount of debris to plug the stent. ${ }^{7}$ The measurement of inner diameter showed heterogeneity along the tube for several stents. These constriction zones on a tube without side holes constitute a significant reduction in the inner diameter of the stent and an increased risk of blockage at this constriction.

Several theories have been put forth to explain the complex interaction that occurs as a microbe approach and then attaches to a surface. ${ }^{5}$ Aydin et al examined 102 patients in whom a ureteral stent was removed after 1 month under aseptic conditions. Bacterial colonization was found in $29.4 \%$ of the stents and the washing fluid used to clean the interior of the stent-produced pathogens in $7.8 \%$ of cases. ${ }^{11}$ This colonization with biofilm formation and maintenance of the infection could promote the encrustation of the stents and their obstruction. ${ }^{5}$ But other causes of obstruction like stent properties, severity of ureter lumen occlusion and fluid mechanics may be involved. ${ }^{1,4,5,10,11}$

In the present study, the analysis of the kinetics of water velocity in the stents showed a progressive increase at the beginning of the compression then a sudden drop until the disappearance of the water flow. Before radial compression, the outline of the surface of the stent lumen was a circle. But any slight crushing of the stent transformed this circle into an ellipse leading to a loss of surface. If the surface area is reduced, the conservation of mass for a fluid in stationary flow then results in an increase in the velocity of the fluid. These wall irregularities induce changes in fluid velocity and could be factors initiating the encrustation of the stent. Indeed, some authors have shown that modifications on fluid flow through a stent caused vortices in the vicinity of the compression of the stent and initialized encrustation. ${ }^{10,12,13}$

Saur et al used a rotating annular reactor with turbulent flow to measure the impact of wall shear stress and evaluate the adhesion in terms of microbiological structures. The number of attached bacteria globally increased with the wall shear stress and as the shear increased, bacteria clusters formed. ${ }^{12}$

De Grazia et al used microfluidic devices (referred to as stent-on-a-chip) with laminar flow at $1 \mathrm{~mL} \mathrm{~m}^{-1}$ and computational fluid dynamic simulations to identify hydrodynamic regions of a stent that are more susceptible to bacterial attachment. They observed that the formation of cavities with low-velocity laminar vortices in areas located in the proximity of a ureteral obstruction caused bacterial attachment in the stented ureter and, the attachment area appeared to be directly proportional to the number of cavities. $^{13}$

Thus, it appears that when ureteral flow passes exclusively through the lumen of the stent, the stent is considered to be a long and thin tube for which inner diameter and stiffness are important. In this study, the data in Table 2 showed that the volumetric flow rate increases with the inner diameter of the stent. With a stent without side holes and an exclusive luminal flow, Kim et al showed that the flow 
remained stable but decreased moderately over the entire length of the stent due to pressure loss. ${ }^{9}$ Some laws of physics may provide new insights to improve reinforced stents for MUO. The Hagen-Poiseuille law, published in 1846 , is a physical law that gives the pressure loss in a fluid in laminar flow passing through a cylindrical tube of constant cross section. This law was applicable to the ureteral stent because the flow was laminar (Low Reynolds number around 10 at $27 \mathrm{~mL} \mathrm{~h}^{-19}$ ), and the size of the lumen was very small compared to the length of the stent. ${ }^{14}$

In the following equation ${ }^{14,15}$ (1) where $\Delta \mathrm{P}, \mathrm{Q}, \mathrm{D}$, $\mathrm{L}$ and $\eta$ represent the pressure loss, the volumetric flow rate, the inner diameter, the length of the tube and the dynamic viscosity, respectively, Poiseuille clearly demonstrated that the volumetric flow rate increases with the inner diameter of the tube. He explained his equation by indicating that if the diameter doubles, sixteen times more fluid will pass through the tube, all other things being equal. ${ }^{14}$ Thus, the inner diameter of the stent is an essential parameter to increase the flow rate.

$$
\begin{gathered}
\Delta \mathrm{P}=\frac{128 \mathrm{~L} \eta \mathrm{Q}}{\pi \mathrm{D}^{4}} \\
\Delta \mathrm{P}=8 \pi \mathrm{Q} \times \frac{\mathrm{L} \times \eta}{\mathrm{S}^{2}}
\end{gathered}
$$

But, equation (2) formulated differently from equation (1) provides other interesting information. First, the pressure loss for a given volumetric flow rate is inversely proportional to the square of the cross-sectional area S. In other words, the slightest reduction in the cross-sectional area is detrimental to the quality of the flow. The preservation of native lumen as a perfect circle is therefore essential. Second, the pressure loss increases with the length of the tube and the viscosity of the fluid.

\section{Length}

The stent in itself represented an obstruction of the flow rate in the ureter ${ }^{10}$ and an excessively long double-pigtail stent crossing the midline of the bladder may represent yet another factor of obstruction. A potential solution could come from the design of the stent itself, by shortening it to the exact length of the ureter as in the method previously described with a silicone end-piece at the bottom of the stent. ${ }^{16}$ This stent was known to decrease stent-related symptoms and may decrease the risk of encrustation as suggested by Hagen-Poiseuille's law.

\section{Viscosity}

In patients apparently healthy with abdominal obesity and aged between 40 and 60, Herranz et al observed that urine viscosity correlated significantly with age. ${ }^{17}$ Moreover, Clavica et al used a silicone ureter and pelvis model and observed that when the ureter was unobstructed, the renal pelvic pressure was stable even at the highest values of fluid viscosity. However, a small increase in urine viscosity associated with ureteral obstruction strongly affected renal pelvic pressure. ${ }^{10}$ Thus, some clinical pathologies with an increase in urine viscosity associated with ureteral obstruction could alter the flow in a ureteral stent and promote the stent encrustation.

Thus, for a better stent patency, the very design of the stent could potentially help via a high degree of stiffness, a wide inner diameter, a short length of the stent, and abundant fluid intake for the patient.

\section{Stent Failure}

Patients with MUO may require lifelong indwelling stent and obstruction is possible despite a selection of stents. Tandem stents or Resonance stents have been developed as alternatives to single ureteric stents. ${ }^{1-3}$ In the present study, the best result against the radial compression was obtained with a tandem of two Teleflex $8 \mathrm{~F}$ stents requiring the highest force $(731.8 \mathrm{~N})$ to stop the water flow and providing the greatest lumen among the stents currently available on the market.

\section{Limitations}

First, the selection of the stents was made with a deliberate bias aimed at replacing an early obstructed stent in a patient with MUO. This study was carried out exclusively in vitro and the results of a clinical study using the same stents could be a better gauge of the importance of stent stiffness and inner diameter of the stent.

Second, this study did not include all commercialized reinforced stents or metal stents or metal meshes. But metal stents cannot be tested with a water flow.

Finally, neither the gravity effect on the urine column circulating in the stent, nor other forces of interaction between the fluid and the walls of the stent were included in the present study.

\section{Conclusions}

The stiffness of the stent appeared to be an important factor to maintain patency with respect to radial compression 
forces but the inner diameter of the stent and its preservation may be essential parameters to increase the volumetric flow rate. Some reinforced stents tested in the present study confirmed that it is possible to combine stiffness and wide lumen. In case of single stent obstruction, the use of tandem provided the best stiffness against radial compression and the greatest lumen. The results of a clinical study using the same stents could be a better gauge of the importance of stent stiffness and inner diameter of the stent.

\section{Abbreviation}

MUO, malignant ureteral obstruction.

\section{Acknowledgment}

I thank Professor Janine Dove-Rumé, English Department at the University of Tours, for re-reading the text.

\section{Author Contributions}

The author made substantial contributions to conception and design, acquisition of data, or analysis and interpretation of data; took part in drafting the article or revising it critically for important intellectual content; agreed to submit to the current journal; gave final approval of the version to be published; and agree to be accountable for all aspects of the work. B Vogt had full access to all the data in the study and takes responsibility for the integrity of the data and the accuracy of the data analysis.

\section{Disclosure}

B Vogt certifies that the only conflict of interest, including specific financial interests and relationships and affiliations relevant to the subject matter or materials discussed in the manuscript is the following: Benoît Vogt is a consultant for Rocamed but states there are no financial competing interests in the manuscript. The author reports no other potential conflicts of interest for this work.

\section{References}

1. Elsamra SE, Leavitt DA, Motato HA, et al. Stenting for malignant ureteral obstruction: tandem, metal or metal-mesh stents. Int $J$ Urol. 2015;22(7):629-636. doi:10.1111/iju.12795
2. Vogt B, Desfemmes FN, Desgrippes A. Improving comfort of patients with ureteral obstruction and malignant disease should be of concern. J Palliat Med. 2016;19(11):1132-1133. doi:10.1089/ jpm.2016.0276

3. Vogt B. Ureteral stent obstruction and stent's discomfort are not irreparable damages. Urol Case Rep. 2018;20:100-101. doi:10.1016/j.eucr.2018.07.025

4. Christman MS, L'esperance JO, Choe CH, Stroup SP, Auge BK. Analysis of ureteral stent compression force and its role in malignant obstruction. $J$ Urol. 2009;181(1):392-396. doi:10.1016/j. juro.2008.08.125

5. Tenke P, Kovacs B, Jäckel M, Nagy E. The role of biofilm infection in urology. World J Urol. 2006;24(1):13-20. doi:10.1007/s00345005-0050-2

6. Schlick RW, Seidl EM, Kalem T, Volkmer B, Planz K. New endoureteral double-J stent resists extrinsic ureteral compression. J Endourol. 1998;12(1):37-40. doi:10.1089/end.1998.12.37

7. Hübner WA, Plas EG, Stoller ML. The double-J ureteral stent: in vivo and in vitro flow studies. $J$ Urol. 1992;148(2 Pt 1):278-280. doi:10.1016/s0022-5347(17)36572-2

8. Stoller ML, Schwartz BF, Frigstad JR, Norris L, Park JB, Magliochetti MJ. An in vitro assessment of the flow characteristics of spiral-ridged and smooth-walled JJ ureteric stents. BJU Int. 2000;85(6):628-631. doi:10.1046/j.1464-410x.2000.00489.x

9. Kim KW, Choi YH, Lee SB, Baba Y, Kim HH, Suh SH. Analysis of urine flow in three different ureter models. Comput Math Methods Med. 2017;2017:5172641. doi:10.1155/2017/5172641

10. Clavica F, Zhao X, ElMahdy M, Drake MJ, Zhang X, Carugo D. Investigating the flow dynamics in the obstructed and stented ureter by means of a biomimetic artificial model. PLoS One. 2014;9(2): e87433. doi:10.1371/journal.pone.0087433

11. Aydin HR, Irkilata L, Aydin M, et al. Incidence of bacterial colonisation after indwelling of double-J ureteral stent. Arch Ital Urol Androl. 2016;87(4):291-294. doi:10.4081/aiua.2015.4.291

12. Saur T, Morin E, Habouzit F, Bernet N, Escudié R. Impact of wall shear stress on initial bacterial adhesion in rotating annular reactor. PLoS One. 2017;12(2):e0172113. doi:10.1371/journal.pone.0172113

13. De Grazia A, LuTheryn G, Meghdadi A, et al. A microfluidic-based investigation of bacterial attachment in ureteral stents. Micromachines (Basel). 2020;11(4):408. doi:10.3390/mi11040408

14. Poiseuille JB [Recherches expérimentales sur le mouvement des liquides dans les tubes de très-petits diamètres]. Royal Printing, 1844. University La Sapienza of Roma (Biblioteca di Ingegneria Civile, Edile e Ambientale); December 2, 2014:111. Available from: https://books.google.fr/books?id=uBN1Q-IRzTMC\&pg. Accessed November 12, 2020. French.

15. Pfitzner J. Poiseuille and his law. Anaesthesia. 1976;31(2):273-275. doi:10.1111/j.1365-2044.1976.tb11804.x

16. Vogt B, New Customized A. Ureteral stent with nonrefluxing silicone end-piece to alleviate stent-related symptoms in malignant diseases. Urology. 2020;137:45-49. doi:10.1016/j.urology.2019.12.022

17. Herranz B, Álvarez MD, Pérez-Jiménez J. Association of plasma and urine viscosity with cardiometabolic risk factors and oxidative status. A pilot study in subjects with abdominal obesity. PLoS One. 2018;13 (10):e0204075. doi:10.1371/journal.pone.0204075 


\section{Publish your work in this journal}

Research and Reports in Urology is an international, peer-reviewed, open access journal publishing original research, reports, editorials, reviews and commentaries on all aspects of adult and pediatric urology in the clinic and laboratory including the following topics: Pathology, pathophysiology of urological disease; Investigation and treatment of urological disease; Pharmacology of drugs used for the treatment of urological disease. The manuscript management system is completely online and includes a very quick and fair peer-review system, which is all easy to use. Visit http://www.dovepress.com/ testimonials.php to read real quotes from published authors. 